\title{
How CRISPR/Cas9 Finds Genetic Targets
}

\author{
A model of facilitated diffusion and the theory of Anderson localization \\ help explain how the Cas9 protein explores DNA in search of its targets. \\ By Christopher Crockett
}

I $\mathrm{n}$ the gene-editing business, CRISPR/Cas9 is all the rage. This technique allows researchers to precisely edit genes cheaply and quickly by exploiting a genetic weapon deployed by the immune systems of bacteria. Now, Simone Pigolotti of the Okinawa Institute of Science and Technology Graduate University, Japan, and colleagues have come up with a simple theoretical model to describe how the CRISPR/Cas9 system may search DNA to find the genes it is looking for [1].

At the heart of CRISPR/Cas9 is a protein dubbed Cas9. It carries a snippet of genetic code and looks for a match in any DNA it encounters. If a match is found, Cas9 severs the DNA right at that spot. Bacteria use this mechanism to identify and destroy viruses that they have encountered in the past. To characterize how Cas9 finds its targets, Pigolotti and collaborators turned to a model of "facilitated diffusion." In this model, Cas9 binds to DNA, slides along a segment, and detaches. Then the protein diffuses through the cell before binding to another region of DNA and starting the cycle anew.

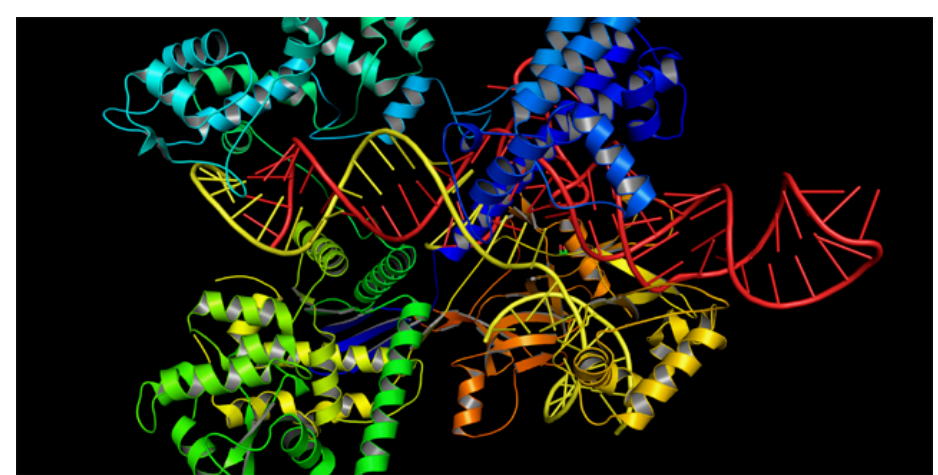

Credit: ibreakstock/stock.adobe.com
The model supports recent experimental results suggesting that Cas9 finds its targets by sliding along the DNA, but it also suggests that the length along which the protein slides is surprisingly short compared with other DNA-sliding complexes. The team also finds an unexpected connection to the theory of Anderson localization, which describes how electrons become bound to certain areas in disordered materials. In this case, Anderson localization helps explain how Cas9 proteins are found to congregate near their genetic targets, elucidating how Cas9 focuses on specific regions within long stretches of DNA.

Christopher Crockett is a freelance writer based in Arlington, Virginia.

\section{REFERENCES}

1. Q. Lu et al., "Search and localization dynamics of the CRISPR-Cas9 system," Phys. Rev. Lett. 127, 208102 (2021). 